http://dx.doi.org/10.23925/1983-3156.2020v22i4p189-203

\title{
Diseño de una actividad didáctica con base en un diálogo entre TAD y APOE \\ Design of a didactic activity based on a dialogue between ATD and APOS
}

\author{
Rita Vázquez ${ }^{1}$ \\ UACM-Universidad Autónoma de la Ciudad de México, México \\ https://orcid.org/0000-0002-6740-235X \\ María Trigueros $^{2}$ \\ ITAM - Instituto Tecnológico Autónomo de México, México \\ https://orcid.org/0000-0001-7527-6704 \\ Avenilde Romo-Vázquez ${ }^{3}$ \\ CICATA-IPN, México \\ https://orcid.org/0000-0002-1364-5997
}

\begin{abstract}
We present the design of a modelling activity based on the dialogue between anthropological theory of the didactic (ATD) and the Action-Process-Object-Schema (APOS) theory. We focus in illustrating the potentiality of this dialogue for the design and analysis of research experiences.
\end{abstract}

Palabras-clave: TAD, APOE, Modelación, Álgebra lineal; Transformación inversa.

\section{Resumen}

Se presenta el diseño de una actividad de modelación a partir del diálogo entre la teoría antropológica de lo didáctico (TAD) y la teoría Acción-Proceso-Objeto-Esquema (APOE). Nos centramos en ilustrar la potencialidad de dicho diálogo para el diseño y análisis de experiencias de investigación.

Keywords: TAD, APOS, Modeling, Linear algebra; Reverse transformation.

\footnotetext{
${ }^{1}$ rita.vazquez@uacm.edu.mx

2 mtriguerosg@gmail.com

3 avenilderv@yahoo.com.mx
} 


\section{Diseño de una actividad didáctica con base en un diálogo entre TAD y APOE}

El trabajo que presentamos continúa la investigación sobre el diseño de actividades didácticas basadas en la modelación matemática para la formación matemática de futuros ingenieros, y tiene como antecedente el artículo de Rita Vázquez, Avenilde Romo-Vázquez, Rebeca Romo-Vázquez y María Trigueros (2016) en el que se presentó un análisis praxeológico de la BSS (Separación Ciega de Fuentes). El análisis permitió identificar la BSS como una praxeología institucional, enmarcada en el Análisis de señales —institución P(DI)—, que actualmente está en una fase de rápida evolución y cuyo origen se remonta a un problema de la neurobiología. En el marco de esta disciplina se sabe que, al mover una articulación, el cerebro recibe información mezclada tanto del cambio de posición de la articulación como de la velocidad al moverla, lo que llevó a la pregunta: ¿es posible imitar la forma en que el cerebro separa estas informaciones? En su planteamiento actual, la BSS consiste en determinar posibles "fuentes" a partir únicamente del conocimiento de mezclas (observaciones). Por ejemplo, separar señales cerebrales (fuentes) de las no cerebrales a partir de un encefalograma (observaciones). Así, el problema de la BSS es un problema de modelado inverso: su punto de partida son los resultados obtenidos en la medición (observaciones), se supone una manera de mezclado y se determinan las fuentes, mediante modelos matriciales y estadísticos.

El análisis de la BSS permitió identificar una praxeología local, que llamaremos $\mathrm{P}_{\mathrm{BSs}}$, en la que se asume que las observaciones son combinaciones lineales de las fuentes (modelo de mezcla instantánea sin ruido) de la forma $T(s)=A s=x$, donde $A$ es la denominada "matriz de mezcla", $s$ representa las fuentes y $x$ las observaciones. Con el objetivo de que la praxeología $\mathrm{P}_{\mathrm{BSS}}$ fuera objeto de enseñanza en un curso de Álgebra Lineal $E(A L)$ en una formación de futuros ingenieros, se pensó e implementó una posible transposición de este conocimiento. Para ello, se reconocieron elementos relevantes en $E(A L)$ como, por ejemplo, el concepto de vector en $R^{n}$, de matriz, de transformación matricial $T(x)=A x$, de transformación matricial inversa $T^{-1}(x)$, de la matriz inversa y del concepto de señal. Para construir la praxeología transpuesta, que llamaremos $\mathrm{P}_{\mathrm{BSSE}} \mathrm{y}$, los conceptos involucrados, se diseñó la actividad didáctica que presentamos en la sección 3 de este trabajo.

Cabe mencionar que además de la praxeología nuclear identificada, el análisis permitió situar la BSS como una praxeología de nivel regional, que es relevante en la ingeniería y tiene aplicaciones 
en dominios fuera de $P(D I)$ (por ejemplo, en la biomedicina o en las telecomunicaciones) y que incluye técnicas y elementos tecnológicos de distintos niveles de complejidad, como se ilustra en la Figura 1. Esta organización praxeológica puede ser la base del diseño de distintas transposiciones para producir actividades didácticas en condiciones institucionales distintas como, por ejemplo, en cursos avanzados de ingeniería en los que se involucren técnicas matemáticas más complejas.

Para el diseño de la actividad y el análisis de su implementación, se utilizó como referente teórico los resultados del diálogo entre la teoría antropológica de lo didáctico (TAD) (Chevallard, 1999) y la teoría Acción, Proceso, Objeto, Esquema (APOE) (Arnon et al., 2014). Esto permitió tomar en cuenta tanto la dimensión institucional de un proceso de estudio planteado a partir de una cuestión problemática — resultado del análisis praxeológico de la BSS-, como la dimensión cognitiva a partir de la metodología de investigación de APOE, de manera integrada. De esta manera fue posible evaluar de forma puntual la construcción de las técnicas y de los elementos tecnológicos presentes en la praxeología adaptada a $E(A L)$, así como el trabajo de los estudiantes a lo largo de la puesta en práctica de la actividad.

\section{Figura 1}

Niveles de organización de la BSS dentro de $P(D I)$.

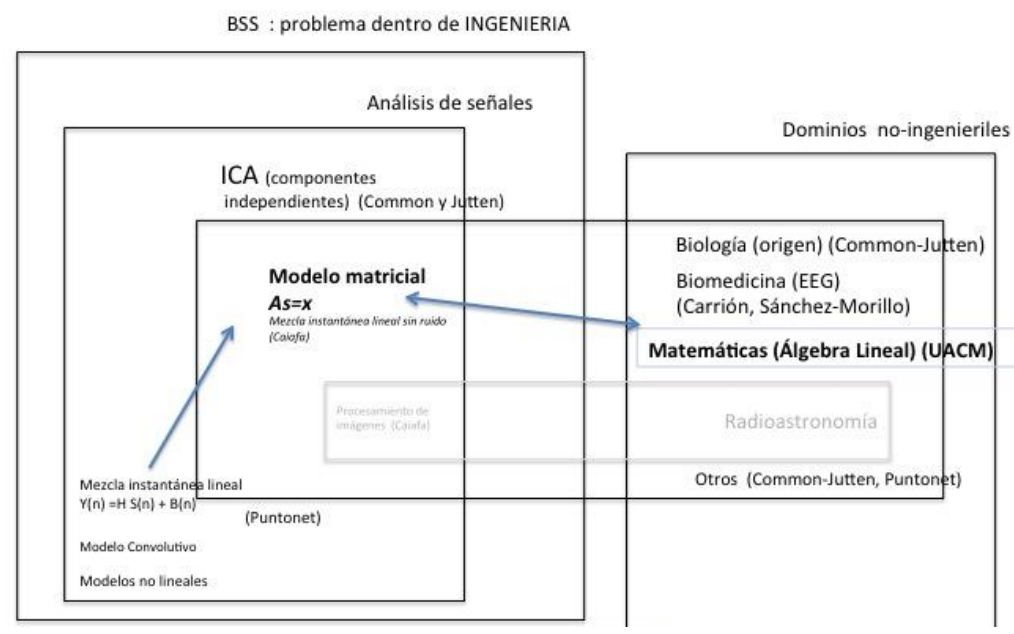

El diálogo entre TAD y APOE

\section{Antecedente: tres modalidades de diálogo}

En el diálogo establecido entre la TAD y APOE, en trabajos previos de Marianna Bosch, Josep Gascón y María Trigueros $(2011,2017)$ se plantearon tres modalidades de diálogo 
entre estas teorías, consideradas como praxeologías de investigación (PI). En la tercera modalidad, el diálogo parte de los componentes técnico y tecnológico de las dos PI, considerando que las construcciones incluidas en la "Descomposición Genética" (DG) de un concepto matemático pueden ser interpretadas de modo que resulten útiles para caracterizar el desarrollo de las técnicas (Bosch, Gascón, \& Trigueros, 2011, p. 97), así como para precisar los grados de completitud de las praxeologías (matemáticas). Los momentos de estudio, que en la TAD definen el proceso de construcción o reconstrucción de una praxeología, pueden conectarse desde el punto de vista institucional con las actividades que en APOE detonan la construcción de conceptos, diseñadas a partir de la DG a través del ciclo ACE, en el marco de la misma teoría. Este ciclo se reformula para proponer una adaptación que se denominó los momentos de estudio del ciclo ACE (A-Actividades, $C$-discusión en clase, E-Ejercicios) (Bosch, Gascón, \& Trigueros, 2011 y 2017).

\section{Un diseño didáctico basado en el diálogo TAD-APOE}

Para diseñar la actividad didáctica se propuso una interpretación de los momentos de estudio del ciclo ACE en la que las fases de distintas actividades funcionan como un detonador para llevar a cabo Acciones o su interiorización en Procesos en forma de tareas matemáticas, que pueden asociarse a los momentos del primer encuentro, de exploración y de trabajo en la técnica, que permitan propiciar la construcción, por parte de los estudiantes genéricos de la institución, de algún elemento inmerso en la praxeología. La construcción de estos elementos — de naturaleza esencialmente matemática — constituye el objetivo del diseño didáctico, y se desarrolla y se evalúa con base en una DG preliminar de dichos elementos. Las fases de discusión en clase $C$ y de ejercicios $E$ propician la reflexión e incluyen actividades dentro de los momentos de trabajo de la técnica, técnico-tecnológico, de institucionalización y de evaluación del ciclo ACE mediante nuevas oportunidades de reflexión para lograr la interiorización de las Acciones en Procesos, la encapsulación de éstos en Objetos o la 
construcción de Esquemas considerados en la DG. Las actividades se desarrollan así a partir de iteraciones progresivas del ciclo, en las que las construcciones alcanzadas permiten llevar a cabo nuevas construcciones. En el desarrollo progresivo del ciclo ACE emergen los momentos de estudio de este, para dar lugar a un proceso de estudio equilibrado, en el que es posible evaluar la DG inicial.

Los antecedentes del diálogo, y el interés de diseñar actividades de modelación que acerquen las instituciones de la práctica con las de la formación matemática desde el primer año, tal y como se propuso con el análisis praxeológico de la BSS, nos llevan a plantear las dos preguntas de investigación que formulamos en los términos siguientes:

(1) ¿Cómo pueden intervenir los resultados del diálogo entre la TAD y la teoría APOE en el diseño de una actividad didáctica basada en modelación?

(2) ¿Es posible que el uso de los resultados del diálogo entre teorías posibilite el aprendizaje de la noción de transformación matricial, tomando en cuenta las condiciones institucionales para su aprendizaje?

\section{EI diseño de la secuencia didáctica: de la BSS al curso de Álgebra Lineal}

Para el diseño de la secuencia se partió de la praxeología "nuclear" que denotaremos como $\mathrm{P}_{\mathrm{BSS}} \mathrm{y}$ que fue identificada a partir del análisis praxeológico. En ella, el tipo de tareas consiste en separar las señales bajo la hipótesis del modelo de mezcla lineal instantánea (Vázquez, Romo-Vázquez, Trigueros, \& Romo-Vázquez, 2016).

Para hacer intervenir la noción de señal se diseñó el "Problema de Espías" que consiste en la siguiente situación: hay cuatro espías que desean descifrar una conversación entre diplomáticos, utilizando como modelo para simular su voz un tipo simple de señales sonoras, conocidas como tonos puros, que matemáticamente se modelan mediante la función de onda $y(t)=a \cdot \sin (2 \pi \omega t)$, donde $\omega$ corresponde a la frecuencia del tono y $a$ es la amplitud de la onda sonora. Así, las fuentes (voces) están representadas por tonos puros de distintas frecuencias, 
denotados $s_{1}, s_{2}, \ldots$ y las observaciones corresponden a registros de la mezcla de voces, por ejemplo, obtenidos con grabadoras posicionadas en distintos puntos de la sala de reunión y denotados como $x_{1}, x_{2}, \ldots$ Las hipótesis del modelo se simplificaron al no considerar el carácter aleatorio de las variables $x_{i}$ y $s_{i}$. Así, el tipo de tarea para $\mathrm{P}_{\mathrm{BSSE}}$ se reformuló sobre cómo averiguar las señales origen, es decir, cómo encontrar $s(t)=\left(s_{1}(t), \ldots, s_{N}(t)\right)^{T}$ a partir de conocer las señales de salida, $\boldsymbol{x}(t)=\left(x_{1}(t), \ldots, x_{p}(t)\right)^{T}$, considerando, como hipótesis, que el proceso corresponde a una transformación lineal, es decir que $T(s)=A s=x$, donde $A$ es una matriz de $N x p$ y donde cada $s_{i}(t)$ es un tono puro de una frecuencia dada. Si bien en la formulación original $A$ es un mapeo no conocido (una hipótesis que en $P(D I)$ lleva a generar técnicas estadísticas para estimar $A^{-1}$ ) es posible operar una transposición para el contexto de tonos puros en la que $A$ puede determinarse a partir de la relación entre la amplitud de la onda sonora y la distancia entre fuentes y observaciones. Para resolver el tipo de tarea $T=\{$ separar tonos puros $\}$ se reconoció una técnica que se basa en el cálculo de la inversa de una transformación matricial que, a su vez, comporta una sub-técnica para calcular la inversa de una matriz. Se hizo un análisis teórico y didáctico de los elementos señal (tono puro), transformación matricial directa, inversa e inversa de una matriz, a partir del cual se propuso una DG preliminar tanto para la construcción de la transformación matricial, como de la matriz inversa. A partir de la DG y, tomando como marco el diálogo entre las dos teorías, se diseñó una actividad didáctica dividida en tres ciclos que se describen a continuación.

\section{Un diseño en tres iteraciones del ciclo ACE}

El primer ciclo parte de un momento del primer encuentro $(M 1)$ con la praxeología de separación de fuentes que tiene por objetivo comenzar a construir el modelo de la mezcla lineal instantánea como un Proceso, de modo que dé lugar a la necesidad de las transformaciones matriciales directa e inversa para poder abordar y resolver el problema de separación. 
Esto se logra a través de dos dispositivos principales: el momento del primer encuentro con la cuestión problemática $Q$ y el uso de las configuraciones de fuentes y observaciones para comenzar a construir el modelo matemático de interés. El momento del primer encuentro se abre con el "Problema de Espías" (presentado en la sección anterior), simulando en el aula la conversación entre tres personas que hablan y registrando la conversación con teléfonos celulares, ubicados en distintas posiciones del salón. Se plantea la siguiente cuestión: ¿cómo se puede identificar a los emisores si solo se cuenta con las grabaciones? El estudio de esta cuestión da pie a un momento exploratorio $(M 2)$ en el que se discute sobre las variables que tendrían lugar en la construcción de una técnica para abordar la tarea, y se propone la realización de tareas en las que se hacen Acciones, de cambio de las configuraciones de fuentes y observaciones con el fin de que los estudiantes interioricen una configuración de fuentes y observaciones como un Proceso de mezcla lineal de fuentes.

El segundo ciclo consolida el momento exploratorio (M2). Se evocan los Procesos conjunto y vector, así como función de una variable para que, al coordinarse con el Proceso mezcla de fuentes se construya la transformación matricial asociada a una configuración como un Proceso.

Esta construcción permite asociar una mezcla de $m$ fuentes produciendo $n$ observaciones al modelo matricial $A s=x$, reconociéndolo como una transformación $T_{A}$ : $R^{n} \rightarrow R^{m}$, la transformación aparece entonces como un elemento tecnológico, y como un Objeto, que permite el trabajo con el modelo. Al disponer de esta construcción surge la necesidad de una técnica para modelar el problema inverso, es decir, separar la mezcla de señales que se traduce en generar una transformación inversa $T_{A}^{-1}: R^{m} \rightarrow R^{n}$ tal que $T_{A}(x)=s$. Al mismo tiempo, surge la necesidad teórica de indagar si existe esa técnica y bajo qué condiciones. Se inicia así la construcción del concepto transformación matricial inversa, a partir de Acciones mediante las cuales los estudiantes exploran la posibilidad de expresar 
matemáticamente un modelo inverso para el modelo de mezcla lineal. A continuación, con la reflexión sobre las Acciones sobre el Objeto Modelo de mezcla y sobre el Objeto Transformación matricial, emerge la necesidad de contar con una técnica para calcular la matriz inversa de A (la matriz de mezcla). Aparecen en el ciclo nuevos momentos de primer encuentro, de exploración y de trabajo en la técnica en los que, con base en la DG de la transformación matricial inversa, se proponen actividades para construir la matriz inversa de $A$ y que da inicio al tercer ciclo de la actividad.

En el tercer ciclo el objetivo es, en primer lugar, construir el concepto matriz inversa y, en segundo, ampliar la construcción de transformación matricial de manera que incluya la existencia de su inversa. En esta parte, el trabajo se separa momentáneamente del modelo original (mezcla de tonos puros) y se concentra en la construcción del objeto matemático (matriz inversa), lo que se pretende lograr como resultado de la coordinación del Proceso producto matriz-vector y el Proceso Sistemas de ecuaciones para calcular los coeficientes de una matriz inversa entrada por entrada - tomando la inversa en el sentido de la matriz que revierte el efecto de la matriz $A$ en la transformación directa-. Este trabajo puede identificarse con un nuevo momento exploratorio y de construcción de la técnica del ciclo ACE (M2 y M3). Se proponen Acciones de cálculo de la inversa entrada por entrada, haciendo variar las matrices (tanto en tamaño como en relación con la dependencia lineal de sus filas) para propiciar la reflexión sobre las condiciones necesarias para que la técnica pueda aplicarse. Se pretende así de explicitar las condiciones que debe cumplir una matriz para ser invertible, lo que se identifica como un momento de producción de discurso tecnológico-teórico (M4). Luego, la discusión en clase y los ejercicios permiten abordar la viabilidad de la técnica, estudiar su alcance y sus limitaciones para construir una ampliación y mejora de la técnica inicial (M5). Esta ampliación, se logra a partir de actividades con base en la DG que proponen Acciones de resolución de sistemas de ecuaciones en la que los vectores $b$ del sistema $A x=b$ son los 
vectores canónicos y que, al coordinarse con el Proceso para calcular la inversa entrada por entrada se interiorizan en la construcción del Proceso asociado al algoritmo de Gauss-Jordan para calcular la matriz inversa y de Procesos asociados a las condiciones de su existencia, lo que proporciona un refinamiento de la técnica inicial. Cuando se han concluido las actividades de construcción de la matriz inversa, se regresa al modelo de transformación matricial y se proponen actividades que coordinan el Proceso matriz inversa con el Proceso transformación matricial para llegar en primer término, a una construcción Proceso de la transformación matricial inversa y, luego, al coordinar éste con el Proceso mezcla de fuentes, construir el Proceso correspondiente al modelo de transformación matricial inversa para una mezcla de sonidos (tonos puros), que permite resolver el problema de separación de fuentes del siguiente modo. El estudiante genérico de la institución distingue de entre un conjunto de posibles configuraciones, aquéllas que llevan a un modelo matricial invertible, lo que permite, a partir de un vector muestra de las observaciones, resolver el problema de separación mediante la transformación matricial inversa.

La secuencia de ciclos que se va construyendo es progresiva, de modo que las construcciones del ciclo anterior sirven como base para propiciar las construcciones del siguiente. El "Problema de Espías" es el punto de partida de la secuencia, cuando se formula mediante el momento del encuentro $(M 1)$ y es también el punto de cierre, cuando se resuelve por completo para configuraciones específicas de fuentes y observaciones. La siguiente tabla muestra, a modo de resumen, las principales características de cada uno de los ciclos a partir de las categorías que han facilitado su análisis. 
Tabla 1

Categorías de análisis para el diseño, a partir de los momentos de estudio del ciclo ACE.

\begin{tabular}{|c|c|}
\hline & Categorías del análisis \\
\hline $\begin{array}{l}\text { Ci } \\
\text { clo } \\
1\end{array}$ & $\begin{array}{l}\text { Momento del encuentro con } \mathrm{P}_{\mathrm{BSS}} \text { a través del "Problema de Espías". } \\
\text { Identificación de los elementos de la praxeología (tarea principal). } \\
\text { Construcción del modelo de la mezcla de señales como un Proceso. }\end{array}$ \\
\hline $\begin{array}{c}\text { Ci } \\
\text { clo } \\
2\end{array}$ & $\begin{array}{l}\text { Construcción de la transformación matricial asociada a una } \\
\text { configuración como un Proceso, que permite asociar una mezcla de } m \\
\text { fuentes produciendo } n \text { observaciones al modelo matricial } A s=x \text {, } \\
\text { reconociéndolo como una transformación } T: R^{n} \rightarrow R^{m} \\
\text { Emergencia de una técnica para calcular } T^{-1}: R^{m} \rightarrow R^{n} \text { tal que } T(x)=s \text {. }\end{array}$ \\
\hline \multirow{2}{*}{$\begin{array}{c}\text { Ci } \\
\text { clo } \\
3\end{array}$} & $\begin{array}{l}\text { Sistematización del cálculo de la inversa a partir de coordinar los } \\
\text { Procesos transformación matricial y sistema de ecuaciones. } \\
\text { Construcción de la matriz inversa como Proceso. } \\
\text { Construcción del algoritmo de Gauss-Jordan (refinamiento de la } \\
\text { técnica). } \\
\text { Construcción de las condiciones para la invertibilidad de } A \text {, a través de } \\
\text { coordinar los Procesos independencia lineal y matriz inversa. }\end{array}$ \\
\hline & $\begin{array}{l}\text { Evaluación: resolver un problema de separación de tonos puros, que } \\
\text { permite dar respuesta a la cuestión generatriz. Coordinación de los } \\
\text { Procesos modelo de mezcla lineal, transformación matricial inversa y } \\
\text { matriz inversa para determinar, dada una configuración, si es posible } \\
\text { revertir la mezcla de señales mediante } s=T^{-1}(x)\end{array}$ \\
\hline
\end{tabular}

\section{Evaluación del diseño}

Dada la imposibilidad de incluir la totalidad de actividades que forman la secuencia en este trabajo, se eligió una actividad del ciclo 1 que llamamos Construcción del modelo matemático para el problema de espías, con el fin de analizar su pertinencia en relación a las preguntas de investigación.

\section{La actividad de Construcción del modelo matemático para el problema de espías}

Como antecedente a esta actividad se generó el momento de primer encuentro con $\mathrm{P}_{\mathrm{BSs}}$, a partir de la simulación con grabaciones y voces descrita anteriormente. A continuación, a partir del trabajo con las configuraciones y los tonos puros, se reformuló la situación inicial proponiendo a los estudiantes el caso en el que hay tres fuentes y cuatro observaciones, como se describe en la configuración de la Figura 2. 


\section{Figura 2}

Una configuración de tres fuentes y cuatro observaciones.

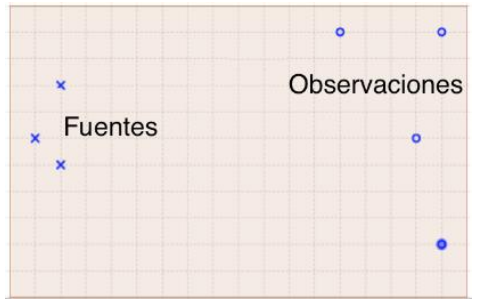

El problema de espías se reformula dando lugar a la cuestión $Q_{1}$ :

$Q_{1}$ : Suponiendo que las voces o fuentes son tres tonos puros de frecuencias $440 \mathrm{~Hz}$, $660 \mathrm{~Hz}$ y $880 \mathrm{~Hz}$, y que se cuenta con las observaciones en un instante.

a) ¿Cómo podría saberse qué tono corresponde a qué fuente?

b) Propongan un modelo para relacionar las variables $x_{i}$ (observaciones) y $s_{i}$ (fuentes).

c) ¿Cuáles son las hipótesis en su modelo?

La pregunta b) se corresponde con un momento de exploración en el que surge la necesidad de generar una técnica inicial que permita resolver $Q_{1}$. La discretización permite al estudiante identificar para un valor de $i$ y un valor de $t_{0}$ específico, los valores de las funciones $s_{i}\left(t_{0}\right)$ y $x_{i}\left(t_{0}\right)$ como números reales, coordinando el Proceso de discretización con el Proceso de función de variable real. Este Proceso se puede coordinar con el Proceso vector haciendo posible identificar la función vectorial $s$ que asigna a cada $t_{0}$ el vector $\left(s_{1}\left(t_{0}\right), s_{2}\left(t_{0}\right), s_{3}\left(t_{0}\right)\right)$. Para lograr la interiorización, se proponen Acciones en las que las preguntas a), b) y c) se plantean de nuevo para configuraciones distintas, haciendo variar el número de fuentes, el número de observaciones y la distancia entre éstas. El germen de técnica inicial (la que permite responder la pregunta $Q_{1}$ ) evoluciona a partir de la interiorización de Acciones para plantear variables y relaciones entre éstas que modelen cualquier configuración de fuentes y observaciones. El trabajo de los equipos se socializa a partir de la discusión en clase, lo que da pie a acordar las 
variables y parámetros del modelo y lleva a establecer una notación común, que será usada por el grupo-clase, por ejemplo:

$$
\begin{aligned}
& s_{i}=s_{i}(t)=\text { fuente } i, \text { para } i=1,2,3 \\
& x_{j}=x_{j}(t)=\text { observación } j, \text { para } j=1,2,3,4 \\
& d_{i j}=\text { distancia de } s_{j} \text { a } x_{i} \text { (en total } 4 \times 3=12 \text { distancias) } \\
& w_{i}=\text { frecuencia del tono fuente } s_{i}, \mathrm{i}=1,2,3
\end{aligned}
$$

Es posible argumentar durante la discusión en grupo a partir de evocar un Esquema de la Física, las dos hipótesis principales del modelo:

(1) La relación inversa entre distancia y amplitud:

$$
x_{1}=a_{11 S_{1}}=\left(1 / d_{11}^{2}\right) \quad s_{1}=\left(1 / d_{11^{2}}\right) \operatorname{sen}\left(w_{1} t\right)
$$

(2) El modelo supone que cada observación es una superposición de las señales fuente:

$$
x_{1}=a_{11} S_{1}+a_{12} S_{2}+a_{13} S_{3}
$$

Se requiere en estas dos hipótesis que los estudiantes hayan construido un Proceso de combinación lineal, que les permita identificar la mezcla lineal de las tres fuentes de la configuración como una combinación lineal de los tres tonos puros, donde los coeficientes estén dados por la relación entre distancia y amplitud. La ecuación descrita en (1) es un modelo conocido, justificado desde la Física y que puede o no ser construido durante la actividad, esto dependerá de los Esquemas previos que los estudiantes tengan con relación al sonido.

En concordancia con la DG preliminar, propuesta para la transformación matricial, se requiere recurrir a una construcción Proceso de sistema de ecuaciones lineales y coordinarla con las construcciones relacionadas con la configuración de fuentes y observaciones, para reconocer el modelo de mezcla como un sistema lineal de $4 \times 3$, con coeficientes reales. Luego de hacer Acciones (primero sobre las dos observaciones restantes $x_{2}$ y $x_{3}$, y luego sobre otras configuraciones), la mezcla lineal de tonos se interioriza como un Proceso, que permite al estudiante, una vez que coordina este Proceso con el Proceso sistema de ecuaciones, construir 
un nuevo Proceso que permite asociar a una configuración de $n$ fuentes y $m$ observaciones, un sistema de $m$ ecuaciones lineales con $n$ variables, en el que los coeficientes de las ecuaciones están dados por los cuadrados de los recíprocos de la distancia entre fuente y observación.

Finalmente, la pregunta c) provoca la reflexión con relación a las condiciones en las que la técnica inicial tiene lugar. Esto corresponde a un momento de trabajo de la técnica, que se ha reconocido como uno que difícilmente se alcanza en enfoques tradicionales de enseñanza, y que como aquí se ejemplifica, puede favorecerse cuando se detona la construcción de Procesos a partir de la reflexión sobre las acciones presentes en la técnica inicial.

Las actividades de los ciclos 2 y 3, diseñadas a partir de la DG, apoyan la construcción de un concepto matemático (la transformación matricial y su inversa) que muestra ser relevante para la formación matemática de futuros ingenieros, como ejemplo de un problema inverso, desde una perspectiva sistémica del producto matriz por vector.

Es importante mencionar que la secuencia completa ha sido implementada en un curso de Álgebra Lineal a lo largo de seis sesiones, y sus resultados, ya analizados, son positivos y serán publicados en un trabajo en elaboración.

\section{Conclusiones}

En relación a la pregunta (1), la aproximación que aquí presentamos cristaliza en una experiencia didáctica específica y muestra las posibilidades prácticas de los resultados teóricos de la interacción entre los enfoques de la TAD y APOE, sin violentar sus supuestos básicos, delineados en el diálogo entre estas teorías (Bosch, Gascón, \& Trigueros, 2011 y 2017). Este proyecto constituye, en este sentido, la primera investigación diseñada completamente dentro de este enfoque de diálogo entre APOS y TAD, basada en modelización, que parte del análisis de una praxeología auténtica dentro de la ingeniería $P(D I)$ y cuyo fin es la construcción de conocimiento matemático en la enseñanza de las matemáticas $E(M)$, al operar una transposición que permite plantear la cuestión problemática inicial para un diseño didáctico. Las actividades 
en el diseño responden a los requerimientos de la DG y de la institución en que se implementa $E(A L)$

Con relación a la pregunta (2) se muestra en la descripción del diseño cómo los resultados del diálogo entre ambas teorías permiten proponer actividades específicas para que los estudiantes logren las construcciones consideradas en la DG, a lo largo de un proceso de estudio bien balanceado. En el primer ciclo, el diseño de actividades se centra en facilitar el momento de exploración del ciclo ACE. En este primer ciclo se incluyen tareas que requieren de Acciones para identificar el papel de las variables que entran en juego en el problema y la construcción de Procesos que conducen al reconocimiento de las configuraciones que posibilitan la construcción de la matriz de mezcla y a la identificación de su papel en la transformación de las señales. Este primer ciclo juega un papel esencial en la construcción del modelo matemático de interés. La reflexión grupal y la discusión en clase permiten hacer las construcciones (Acciones, Procesos, Objetos) que sustentan el aprendizaje de estos conceptos matemáticos, a la vez que permiten la puesta en práctica de habilidades que pueden desarrollarse a través de la modelización. Aunque en este artículo sólo se reportan resultados sobre el ciclo 1 de la secuencia didáctica, constatamos que en las actividades de los ciclos 2 y 3 pudo observarse la emergencia de los momentos exploratorio y de desarrollo tecnológico enmarcando la construcción de un concepto matemático (la transformación matricial y su inversa) que muestra ser relevante para la formación matemática de futuros ingenieros (como ejemplo de un problema inverso), desde una perspectiva sistémica del producto matriz por vector. En las tareas propuestas para abrir el momento del primer encuentro con el cálculo de la matriz inversa, los estudiantes lograron interiorizar Acciones para iniciar una técnica de cálculo de $A^{-1}$ a partir de resolver sistemas de ecuaciones relacionados con una configuración específica de fuentes y observaciones. Luego, en un nuevo momento exploratorio se logró hacer un refinamiento de la técnica inicial, produciendo una técnica más eficiente, que es el 
algoritmo de Gauss-Jordan. Los momentos (M4) y (M5) aparecieron para establecer las limitantes de la técnica y su utilidad para resolver el problema de separación revirtiendo el efecto de la transformación matricial, así como para identificar las condiciones que debe cumplir una matriz para ser invertible. Así, los estudiantes lograron en su mayoría una construcción Proceso que permite calcular la inversa de una matriz a partir de la DG propuesta y pudieron utilizar esta construcción para resolver un problema de separación de tonos puros. Esta perspectiva da un giro a la tradición didáctica de la enseñanza del algoritmo para calcular la matriz inversa, resaltando el enfoque funcional y conectando las construcciones matemáticas con Esquemas no matemáticos, como los relacionados con el sonido.

\section{Referencias}

Arnon, I., Cottrill, J., Dubinsky, E., Oktaç, A., Roa Fuentes, S., Trigueros, M., \& Weller, K. (2014). APOS Theory. A Framework for Research and Curriculum Development in Mathematics Education. Nueva York: Springer-Verlag.

Bosch, M., \& Gascón, J. (2014). Introduction to the Anthropological Theory of the Didactic (ATD). En A. Bikner-Ahsbahs \& S. Prediger (Eds.), Networking of theories as a Research Practice in Mathematics Education (pp. 67-83). Cham: Springer.

Bosch, M., Gascón, J., \& Trigueros, M. (2011). Tres modalidades de diálogo entre APOS y TAD. En Bosch, M. (Ed.), Un panorama de la TAD (pp. 77-116). Bellaterra, España: Centre de Recerca Matemática.

Bosch, M., Gascón, J., \& Trigueros, M. (2017). Dialogue between theories interpreted as research praxeologies: the case of APOS and the ATD. Educational Studies in Mathematics, 95(1), 3952

Chevallard, Y. (1999) L'analyse des pratiques enseignantes en théorie anthropologique du didactique. Recherches en Didactique des Mathématiques, 19(2), 221-266.

Vázquez, R., Romo-Vázquez, A., Romo-Vázquez, R., \& Trigueros, M. (2016). La separación ciega de fuentes: un puente entre el álgebra lineal y el análisis de señales. Educación Matemática, 28(2), $31-57$. 